A. А. Евтифеев, Ю. Л. Ломухин. Обратное кроссполяризационное отражение в однородных граничащих средах

УДК 537.87

DOI 10.18101/2306-2363-2019-1-17-23

\title{
ОБРАТНОЕ КРОССПОЛЯРИЗАЦИОННОЕ ОТРАЖЕНИЕ В ОДНОРОДНЫХ ГРАНИЧАЩИХ СРЕДАХ
}

\author{
(C) А. А. Евтифеев \\ инженер, \\ лаборатория радиозондирования природных сред, \\ Институт физического материаловедения СО РАН \\ 670047, Улан-Удэ, ул. Сахъяновой, 8 \\ E-mail: lexse7en@yandex.ru
}

(C) Ю. Л. Ломухин

доктор физико-математических наук,

лаборатория радиозондирования природных сред,

Институт физического материаловедения СО РАН

670047, Улан-Удэ, ул. Сахъяновой, 8

E-mail: lomukhin_yuriy@mail.ru

Предложен флуктуационный механизм возбуждения кроссполяризационного отражения в поглощающих однородных средах. При этом средняя интенсивность преломленного поля и средняя интенсивность теплового излучения становятся равными. Показано, что кроссполяризационное обратное отражение возникает, как результат рассеяния (отражения) флуктуационных колебаний на тепловых флуктуациях. Установлен эффект образования приповерхностного слоя, в котором формируется как когерентное, так и некогерентное обратное отражение.

Ключевые слова: кроссполяризационное отражение; флуктуационный механизм; тепловые флуктуация; тепловое излучение; обратное отражение; однородные среды; преломленные поля.

Для цитирования:

Евтифеев А. А., Ломухин Ю. Л. Обратное кроссполяризационное отражение в однородных граничащих средах // Вестник Бурятского государственного университета. Химия. Физика. 2019. Вып. 1. С. 17-23.

Кроссполяризационное обратное отражение - это отражение радиоволн с поляризацией отличной от поляризации падающего на границу раздела излучения.

Известно кроссполяризационное обратное рассеяние при облучении случайно-неоднородных сред, представляющих собой коллоидные суспензии - растворы, содержащие частицы [1]. Механизм деполяризации отраженных волн естественно связывают с многократным рассеянием на частицах. В работе покажано, что кроссполяризационное обратное отражение существует в однородных средах, которые допускают теоретическое описание с использованием комплексных диэлектрических проницаемостей. Это сплошные твердые, жидкие, газообразные среды, в которых выполняется условие $\lambda>a$, где $\lambda-$ длина волны падающего на границу раздела излучения, $a-$ среднее межатомное расстояние. 
Некогеретное обратное отражение при нормальном падении волн на границу раздела. Пусть на границу «воздух - полубесконечная поглощающая среда» падает нормально к границе плоская гармоническая волна, рис. 1.

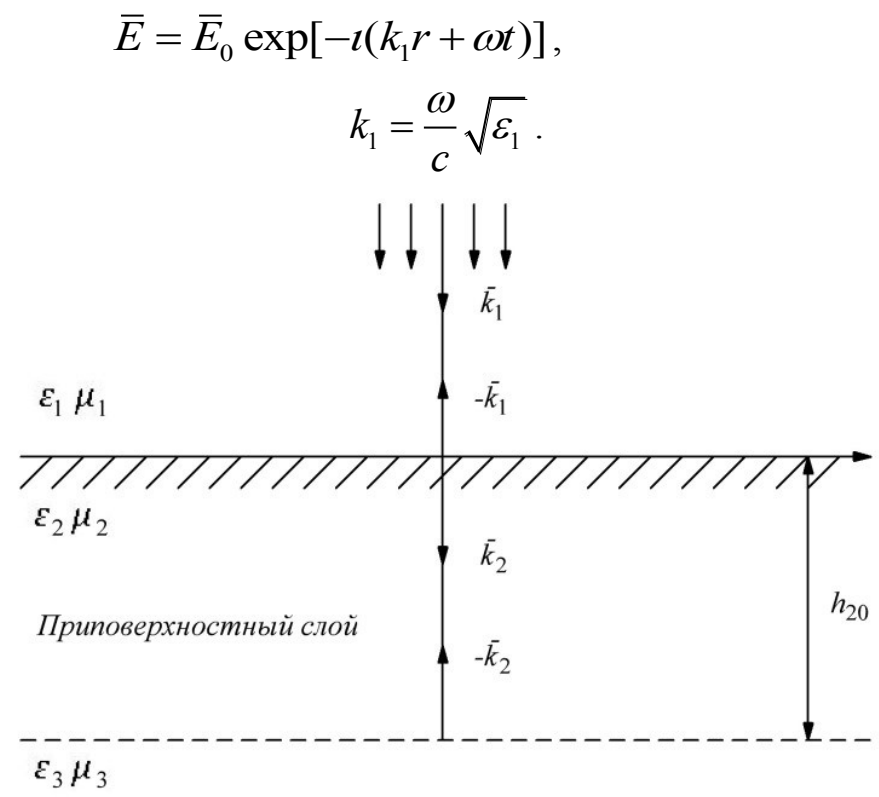

Рис. 1. Схема падения волн на границу раздела

Среды характеризуются параметрами $\varepsilon_{1}=\varepsilon_{1}^{\prime}-\imath \varepsilon_{1}^{\prime \prime}, \quad \varepsilon_{1}^{\prime \prime}<<\varepsilon_{1}^{\prime}, \quad \varepsilon_{2}=\varepsilon_{2}^{\prime}-\imath \varepsilon_{2}^{\prime \prime}$, $\varepsilon_{3}=\varepsilon_{3}^{\prime}-\imath \varepsilon_{3}^{\prime \prime}, \mu_{1}=\mu_{2}=\mu_{3}=1 . \varepsilon_{1}, \varepsilon_{2}, \varepsilon_{3}$ зависят только от частоты.

Прошедшая через границу раздела волна, своим полем вызывает поляризацию во второй среде - возбуждает вторичные источники. В результате взаимодействия полей вторичных источников и прошедшей волны формируются преломленная и встречная волны [2]. При этом интенсивность встречной волны пропорциональна интенсивности преломленной волны. Во второй среде, наряду, с когерентной преломленной волной и когерентной встречной волной возбуждается флуктуационные колебания со случайными направлениями поляризации [3]. В результате преломления на границе раздела встречных волн, в первой среде распространяются как когерентные, так и некогерентные обратные волны в направлении строго противоположном падающему излучению.

Уточним механизм обратного когерентного и некогерентного отражения. Как известно, в любой среде при абсолютной температуре отличной от нуля существует собственные тепловые излучения со случайным направлением поляризации. Поскольку интенсивность преломленной волны уменьшается при распространении вглубь среды, то при некоторой глубине $h_{20}$, рис. 1 , интенсивность преломленной волны сравнивается со средней интенсивностью теплового излучения. Следовательно, вблизи границы $h_{20}$ когерентная преломленная волна трансформируется в некогерентное излучение. Таким образом, до глубины $h_{20}$ 
A. А. Евтифеев, Ю. Л. Ломухин. Обратное кроссполяризационное отражение в однородных граничащих средах

существует когерентная преломленная волна, после перехода в более глубокую область её поляризация становится случайной. Следовательно, на глубине $h_{20}$ образуется граница, при переходе которой интенсивность преломленной волны уменьшается в Р раз, где $\mathrm{P}$ - закон распределения поляризации волн теплового излучения. Такая же трансформация вблизи границы $h_{20}$ происходит с кроссполяризационной компонентой флуктуационного поля.

Из сказанного следует, что при облучении границы раздела сред имеет место два эффекта: во-первых, возбуждается кроссполяризационное обратное отражение, во-вторых, образуется приповерхностный слой толщиной $h_{20}$. В данном слое формируется как когерентное, так и кроссполяризационное обратное отражение.

Определим глубину приповерхностного слоя, для этого напишем равенство

$$
\mid\left(1-\left.V_{1,2}^{P S}(\Delta, 0)\right|^{2} \exp \left[-2 \operatorname{Im}\left(k_{2}\right) h_{20}=n_{1}^{2}\left|1-V_{1,2}^{P S}(\Delta, 0)^{2}\right|\right.\right.
$$

где $V_{1,2}^{P S}(\Delta, 0)=V_{1,2}^{P S}(0) \exp \left(-2\left(k_{1} \Delta\right)^{2}\right), \quad V_{1,2}^{P S}(0) \quad$ _ коэффициент Френеля, $\exp \left(-2\left(k_{1} \Delta\right)^{2}\right)$ - фактор Релея [4], $\Delta$ - средне квадратичное отклонение неровностей границ раздела, $k_{2}=\frac{\omega}{c} \sqrt{\varepsilon_{2}}, n_{1}^{2} \approx \varepsilon_{1}$.

В (2) с левой стороны представлено выражение для ослабления интенсивности преломленной волны прошедшей от границы раздела до глубины $h_{20}$. Справа - интенсивность теплового излучения в среде. Из (2) определяем $h_{20}$ :

$$
h_{20}=h_{20}^{P S}=\frac{1}{2} \frac{1}{\operatorname{Im}\left(k_{2}\right)} \ln \left|\frac{1+V_{1,2}^{P S}(0)}{1-V_{1,2}^{P S}(0)}\right|, \operatorname{Im}\left(k_{2}\right)>0 .
$$

Значение $h_{20}^{P S}$ есть не что иное, как глубина проникновения когерентной волны при облучении границы поглощающей среды. В случае проводящей среды (3) совпадают с известным значением скин - слоя [5].

Учитывая, что интенсивность кроссполяризационной обратной волны пропорциональна интенсивности преломленной и то, что обратное отражение формируется в приповерхностном слое глубиной $h_{20}^{P S}$, используя флуктуационнодиссипационную теорему [6], запишем среднюю интенсивность обратного кроссполяризационного отражения

$$
<\left(\bar{E} \bar{E}^{*}\right)>_{\perp}=A \frac{\pi}{2} \theta(\omega, T) W_{1,2}^{P}(\Delta, 0) W_{1,2}^{P}(\Delta, 0)^{*} W_{1,2}^{S}(\Delta, 0) W_{1,2}^{S}(\Delta, 0)^{*},
$$

где $\frac{\pi}{2} \theta(\omega, T)$ - функция Планка, индексы р и s означают соответственно поляризацию магнитного вектора и поляризацию электрического вектора, Т — абсолютная температура.

$$
W_{1,2}^{P S}(\Delta, 0)=\frac{V_{1,2}^{P S}(\Delta, 0)+V_{2,3}^{P S}(\Delta, 0) \exp \left(-2 i k_{2} h_{20}\right)}{1+V_{2,3}^{P S}(\Delta, 0) V_{1,2}^{P S}(\Delta, 0) \exp \left(-2 i k_{2} h_{20}\right)}-
$$

коэффициент отражения от слоя [7]. 
В (4) $V_{23}^{P S}(\Delta, 0)$ - коэффициент отражения на границе $h_{20}^{P S}$. В силу закона сохранения энергии должно выполняется условие:

$$
<\left(\bar{E} \bar{E}^{*}\right)>_{\perp}\left|\left(1-V_{2,3}^{P S}(\Delta, 0)\right)\right|^{2}=P^{2}<\left(\bar{E} \bar{E}^{*}\right)>_{\perp}
$$

Физический смысл равенства (5) состоит в том, что волна с определенной поляризацией пройдя через границу $h_{20}^{P S}$ трансформируется в излучение со случайной поляризацией, направление которой распределено в интервале $0 \div 4 \pi$ с вероятностью $P$. При этом интенсивность волны с первоначальной поляризацией уменьшается в $P$ раз. Другими словами в данном случае проявляется эффект перехода энергии во флуктуации. Из (4) следует, что $V_{2,3}(\Delta, 0) \approx 1-P$.

Согласно флуктуационно-диссипационной теореме [6], средняя интенсивность теплового излучения может быть представлена как

$$
<\left(\bar{E} \bar{E}^{*}\right)>_{\text {mепл }}=\frac{\pi}{2} \theta(\omega, T) n_{1}^{2}\left(1-W_{1,2}^{P S}(\Delta, 0)^{2}\right) .
$$

В результате трансформации на границе $h_{20}^{P S}$ средняя интенсивность (4) сравнивается с величиной $<\left(\bar{E} \bar{E}^{*}\right)>_{\text {тепл }} P$. Отсюда определим множитель $A$.

$$
A=\frac{n_{1}^{2}\left(1-W_{1,2}^{P S}(\Delta, 0)^{2}\right) P}{W_{1,2}^{P}(\Delta, 0) W_{1,2}^{P}(\Delta, 0)^{*} W_{1,2}^{S}(\Delta, 0) W_{1,2}^{S}(\Delta, 0)^{*}}, P<1 .
$$

Тогда средняя интенсивность кроссполяризационного обратного отражения может быть представлена в виде

$$
<\left(\bar{E} \bar{E}^{*}\right)>_{\perp}=P n_{1}^{2}\left(1-W_{1,2}^{P S}(\Delta, 0)^{2}\right) .
$$

Если допустить равномерное распределение направлений поляризации в интервале $0 \div 4 \pi$, то $P=\frac{1}{4 \pi}$. В представленной методике расчета кроссполяризационного обратного отражения необоснованным является выбор закона распределения $P$. Для его уточнения можно использовать результаты экспериментальных исследований.

\section{Экспериментальные исследования}

Измерения средней интенсивности кроссполяризационного обратного отражения проводились на созданном измерительном комплексе, рис. 2.

Комплекс состоит из двух параболических антенн, у которых имеется устройства для изменения направления поляризации при излучении и приеме электромагнитных волн.

Антенны располагались на общей несущей. Расстояние между центрами антенн выбиралось с учетом достаточной электромагнитной развязки.

Одна антенна подсоединялась к генератору непрерывного излучения частотой 10 ГГц, другая — к измерительному приемнику ПК7-8. 
A. А. Евтифеев, Ю. Л. Ломухин. Обратное кроссполяризационное отражение в однородных граничащих средах

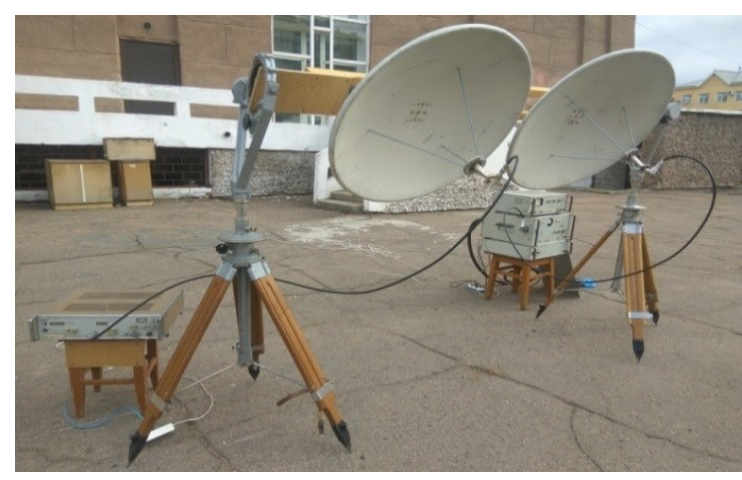

Рис. 2. Измерительный комплекс

Методика измерений сводилась к следующему: вначале антенны ориентировались строго в зенит при VV и VH поляризациях, в обоих случаях измерялся уровень связи между антеннами, который составлял $\simeq$. Затем антенны ориентировались строго на поверхность асфальтового покрытия, и при VV и VH измерялся уровень обратного отражения. Поверхность асфальтового покрытия шероховатая.

Результаты нескольких измерений приведены в таблице.

Таблица

\begin{tabular}{|c|c|c|c|}
\hline № изм. & $\begin{array}{c}\text { Уровень отражения при } \\
\text { VV-поляризации, дБ }\end{array}$ & $\begin{array}{c}\text { Уровень отражения при } \\
\text { УН-поляризации, дБ }\end{array}$ & $\begin{array}{c}\text { Разница } \\
\text { между VV и VН, дБ. }\end{array}$ \\
\hline 1 & 36 & 28 & 8 \\
\hline 2 & 40 & 26 & 14 \\
\hline 3 & 39 & 30 & 8 \\
\hline 4 & 36 & 28 & 6 \\
\hline 5 & 36 & 30 & 5 \\
\hline 6 & 32 & 27 & 15 \\
\hline 7 & 34 & 19 & 16 \\
\hline 8 & 35 & 19 & 20 \\
\hline 9 & 35 & 21 & 18 \\
\hline 10 & 38 & 18 & 12 \\
\hline 11 & 36 & 18 & \\
\hline Сред. Зн. & 36 & 24 & 14 \\
\hline
\end{tabular}

Из нее следует, что средняя величина разности уровней при VV и VH поляризациях отраженного поля составляет $12 \partial Б$, то есть кроссполяризационное отражение на $12 \partial Б$ слабее отражения при кополяризации. Следует отметить, что измерения проведены со значительной погрешностью из-за несовершенства измерительного комплекса, из-за влияния отражений от окружающих объектов и т.д. Поэтому результаты следует считать предварительными. Сравним измеренные и теоретические данные. Для этого воспользуемся формулой (8). В нашем случае $\left(k_{1} \Delta\right)^{2}>>1$, поэтому в (8) можно заменить $W_{1,2}^{P S}(\Delta, 0)$ на $V_{1,2}^{P S}(\Delta, 0)$. Диэлектрическая проницаемость асфальта на частоте 10 ГГц $\varepsilon_{2}=4,3-\imath 0,1$, тогда 
$\left|V_{1,2}^{P S}(\Delta, 0)\right|^{2}=0,4$. Подставляя в (8) и, выражая средний уровень кроссполяризационного отражения в $\partial Б$, получим $\sigma_{V H}(\Delta, 0)=10 \log \frac{1}{4 \pi}(1-0,4)=-13,21 \partial Б$. Уровень обратного отражения при VV поляризации $\sigma_{V V}(\Delta, 0)=10 \log 0,4=-3,97 \partial Б$.

Разница $\sigma_{V V}(\Delta, 0)$ и $\sigma_{V H}(\Delta, 0)$ составляет $9,24 \partial Б$. С учетом погрешностей, результаты измерений и расчётов качественно согласуются между собой.

\section{Заключение}

Предложен физический механизм возбуждения кроссполяризационного обратного отражения включающий возбуждения флуктуационных встречных полей и отражение от границы, где средняя интенсивность преломленного поля и средняя интенсивность теплового излучения становится равными. Установлен эффект образования приповерхностного слоя, в котором формируется как когерентное, так и некогерентное обратное отражение.

\section{Литература}

1. Кузьмин В. Л., Меглинский И. В. Обратное рассеяние света с линейной и круговой поляризациями в случайно-неоднородных средах // Оптика и спектроскопия. 2009. - Т. 106, № 2. - С. 294-305.

2. Lomukhin Yu. L., Atutov E. B., Butukhanov V. P. Backward reflection in the Fresnel problem // JEEE Transaction on Antennas and Propagtion. — 2018. — V. 66, № 4. - P. 18381845 .

3. Ландау Л. Д., Лифшиц Е. М. Электродинамика сплошных сред. - М.: Наука. Главная редакция физико-математической литературы, 1982. - 620 с.

4. Басс Ф. Г., Фукс И. М. Рассеяние волн статистически первой поверхности. - М.: Наука, 1972. - 191c.

5. Тамм И. Е. Основы теории электричества. - М.: Наука. Главная редакция физико-математической литературы. - $614 \mathrm{c}$.

6. Рытов С. М., Кравцов Ю. А., Татарский В. И. Введение в статистическую радиофизику. 4. II. Случайные поля. - М.: Наука. Главная редакция физико-математической литературы, 1978. - $463 \mathrm{c}$.

7. Бреховских Л. М. Волны в слоистых средах. - М.: Наука, 1973. — 341 с.

\section{REVERSE CROSS-POLARIZATION REFLECTION IN HOMOGENEOUS LIMITING ENVIRONMENTS}

\section{A. A. Evtifeev}

Engineer

Laboratory of Radio Sounding of Natural Environments

Institute of Physical Materials Science SB RAS

670047, Ulan-Ude, Sakhyanova Str., 6

E-mail: lexse7en@yandex.ru 

однородных граничащих средах

Yu. L. Lomukhin

Doctor of Physical and Mathematical Sciences

Laboratory of Radio Sounding of Natural Environments

Institute of Physical Materials Science SB RAS

670047, Ulan-Ude, Sakhyanova Str., 6

E-mail: lomukhin_yuriy@mail.ru

The fluctuation mechanism of cross-polarization reflection excitation in absorbing inhomogeneous media is proposed. The average intensity of the refracted field and the average intensity of thermal radiation become equal. It is shown that the cross polarization inverse reflection occurs as a result of the scattering (reflection) of fluctuations in thermal fluctuations. The effect of formation of a near-surface layer in which both coherent and incoherent back reflection is noted.

Keywords: cross polarized reflection; fluctuation mechanism; thermal fluctuation; thermal radiation; back reflection; homogeneous medium; refracted field. 